\title{
Extraction of Keyterms by Simple Text Mining for Business Information Retrieval
}

\author{
Xiangzhu Gao and San Murugesan \\ Southern Cross University \\ Lismore, NSW 2480, Australia \\ \{xgao,smurugesan\}@scu.edu.au
}

\author{
Bruce Lo \\ University of Wisconsin-Eau Claire \\ Eau Claire, WI 54702-4004, USA \\ lobw@uwec.edu
}

\begin{abstract}
Much of business information is text and the information is subject to frequent changes. The use of efficient and effective mechanisms to retrieve required business information is a key to business success, and automated processing of text to extract keyterms is an essential component of such an information retrieval (IR) system. Traditional text processing methods based on complex linguistic or statistic techniques are not efficient in dealing with frequently changing business information and do not necessarily provide satisfying $I R$ results. We propose a simple method to extract important terms (keyterms) from text for application in different aspects of IR and show through experimentation that its performance is comparable to or better than complex methods.
\end{abstract}

\section{Introduction}

With the progress of electronic commerce and emergence of information-intensive business operations, the ability to retrieve information of specific interest effectively and efficiently is of economic importance [1] to a large number of enterprises. Much of business information is text and text mine of many firms is an unstructured collection of documents [2]. Information search in the mine is a young and fertile area of research and practice [3]. Although most of enterprise information is available on the Web, there is little to aide people in search of the information. Finding relevant information is a major problem, which is partly due to the lack of common architecture for enterprise Web sites. To assist users in IR, most business Web sites have a dedicated search engine integrated with the rest of the system and this search engine is expected to perform better than general-purpose Web search engines.
In essence, all search engines are index-based. They index Web pages and try to match a user query with index terms to determine the sources or pages of relevant information. To resolve the problem of vocabulary mismatch between queries and the index, a thesaurus or taxonomy is often used. However, the thesaurus is domain-specific and often needs the intervention of professional experts. In enterprise Web sites, data are updated frequently and hence indexing processes that require frequent human intervention are not well-suited for enterprise information search. Thus, for an effective enterprise search, we need automated indexing methods that quickly index frequently changing information and retrieval schemes that do not rely on thesaurus such as that produced by human experts.

Although natural language processing (NLP) techniques have been proposed for tagging contents of Web pages and learning techniques have been used for determining index terms, they are complex techniques and are not appropriate for enterprise systems. Nevertheless, techniques such as text mining that start with the simple text processing can be used for automated indexing without having to face the full challenge of natural language understanding by machines [4].

In this paper, we propose a simple method of text mining to automatically index Web pages and to use the index as a thesaurus dynamically in the IR process. In the proposed method, keyterms are extracted from text based on simple syntactic rules, geographic layout of document, occurrence of terms and co-occurrence of related terms. The extracted keyterms represent the text content and are often semantically coherent to each other. They can be directly used as index terms. Unlike other methods, which prescribe the number of words in a term, the proposed method allows extracted terms to include any number of words. This will satisfy the natural use of terms in text without including surplus addition or breaking semantic integrity. Because of the semantic coherency of the terms, a sufficiently large set 
of terms can be used for solving the vocabulary problem by partial analysis of the terms. In this paper, we shall focus on extracting the keyterms by means of simple text mining.

This paper is organized as follows. In the second section, we shall describe the background of this work. In the third section, we shall discuss the extraction of terms for indexing and other purposes. In the fourth section, we shall describe the experiment that validates the proposed method and present the evaluation results. The last section will make conclusion and discuss further work.

\section{Background}

An IR system performs matching function [2]. IR consists of two key processes: document storage and document retrieval. In the storage process, an IR system defines a collection of documents, specifies the manipulation of the documents, and represents the documents with an index. In the retrieval process, a user specifies an information requirement, which is then manipulated by the system and represented with a query in a certain format according to the retrieval strategy of the system. The query is then compared with the index to identify documents that are relevant to the query, and relevant documents are retrieved and presented to the user.

Three key issues must be considered in IR. Firstly, the choice of appropriate terms is a challenge [5] for both index creation and query generation. A term can be a single word or a multi-word phrase. Simple comparison between words occurring in a query and the index will not be adequate in all cases. It produces high recall but low precision as the concept difference of a word is ignored by the emphasis on matching chance. Phrase comparison is also problematic because of the different combinations of words that represent the same or similar meanings in a query and the index. It produces high precision but low recall as the same concepts may be missed by the narrow focus on a specific word order. Most experiments show that using phrases in IR obtains inconsistent results [6]. Another question in using phrases is how many words in a phrase. Many experiments use two-word phrases [7, 8, 9], while some others use n-word phrases, where $n$ is normally 1,2 , or 3 .

Secondly, a fundamental problem that hinders a successful retrieval is term mismatch or vocabulary problem [10]. Frequently, terms used by users do not match those that represent the same or similar meanings in documents. A common solution to this problem is to create a thesaurus, which coordinates the usage of the query terms and index terms. However, thesauri are domain-specific and collection-dependent, and often need intervention of professional experts. Thesauri of many kinds have been constructed, often tailored to particular topic areas. Scores of professional associations and government agencies have developed and applied ANSI standard thesauri when indexing specialised content in their field [5]. However, it has not been possible to obtain reliable improvements in retrieval effectiveness by using thesauri in heterogeneous text environments.

Thirdly, without detailed knowledge of document collection and retrieval environment, users find it difficult to formulate appropriate queries. In some situations, users do not know what they really want to search for [11]. In other situations, they cannot specify precisely their information requirement [12]. However, most users can explain their requirements with reference to a specific example. Therefore, some IR systems incorporate the technique of case-based reasoning (CBR), which formulates a query by analysing examples of relevant documents. A major task of CBR is to extract terms from the examples for the generation of a query.

Concerning the above three issues, an essential problem is the determination of terms for index and/or query based on text mining, the automatic or partially automatic processing of text [2]. Most research follows a linear sequence to determine the terms. In the first step, insignificant words are filtered out. Lexical analysis indicates that the most frequent words in English are grammatical factors. Although vital to syntax, they are not significant in discriminating the document contents. Such common words are referred to as stop words. Stop words tend to diminish the impact of frequency difference among less common words, and result in unproductive processing if left in the text.

In the second step, terms are determined by linguistic analysis of text. The basic procedure of the analysis includes recognising certain types of term (words or phrases), normalising the terms to get a canonical form, and finding the relationship among the terms. Most systems are restricted to noun phrases. The assumption behind the restriction is that an occurrence of the noun phrase in a document usually provides much more evidence than other phrases for the concept of the text [13]. A tool such as the part-of-speech tagger can facilitate the recognition of different types of sentence constituents.

The third step is the statistical approach involving various counting techniques. Distinct terms have varying relevance when used to describe text contents [14]. This effect is captured through the assignment of numerical weights to each term by the observation of the value of a random variable (for example, term occurrence frequency) [15]. This weight quantifies the 
importance of the term for describing the semantic contents of the text.

In the above process, complex methods of NLP and statistical analysis were used with limited success [16]. Automated NLP may not suitable in IR because of the linguistic variations. Statistics of phrases may be wrong as a phrase is statistically correlated with its components and phrases have a very different distribution over documents from that of words [6]. The cost of complex methods does not correspond with a guarantee of improving results [17].

In this paper, we propose a method to extract important terms from text based on very simple syntactic rules, geometric layout of documents, frequency counting and relationships between related terms. Together with a method of measuring space in retrieval process [18], this term extraction method performs well with respect to effective document retrieval [19].

\section{Extraction of keyterms}

Extraction of keyterms from a document by text mining consists of two subprocesses: text segmentation by identifying stop terms and ranking (ordering) the terms in descending order with respect to their significance in describing the content of a document or a Web page.

\subsection{Stop term}

It is common to define a stop word list for the removal of semantically insignificant words before text analysis. Articles, prepositions and conjunctions are natural candidates. Other words are also included in the list, if they tend to appear in too many documents or are too common in the user's information requirements. In practice, stop word lists vary in size from around 15 to over 500 words, with most containing around 250 to 300 words.

We defined a stop term list [20], which includes common words, phrases and characters. The following are the principles for the construction of the stop term list.

- An article is an explicit indication of a noun word or phrase, which provides much more evidence than other phrases for the concept of the text [13]. An article delimits noun word or phrase and therefore is a stop term.

- Like an article, a preposition is another indication of a noun or noun equivalent. Propositions are stop terms.
- A conjunction joins together sentences, clauses, phrases, or words. It does not play a semantic role in text. Conjunctions are stop terms.

- A pronoun functions as a substitute for a noun or noun phrase previously specified, or understood from the context. However, it is treated as a stop word for three reasons: the substantive it substitutes for accompanies it near by; it is complex to determine the associated substantive automatically; and the disadvantage caused by determination error may surpass the benefit of the determination. Pronouns are taken as stop terms.

- Some adjectives and adverbs, such as 'some', 'many', 'very' and so on, are too common and ambiguous to represent document topics. These adjectives and adverbs are treated as stop terms.

- The similar situation occurs to some verbs such as all forms of the verb 'be'. Our stop term list includes some common and ambiguous verbs.

- An auxiliary verb, such as 'may' 'can', or 'will', accompanies the main verb in a clause and helps make distinctions in mood, voice, aspect, and tense, but not in contents. Therefore they are stop terms.

- Interjections are a type of stop terms. But we do not include them in our stop term list as they don't often occur in business documents.

- Some words can be syntactically common when they are used in idioms, although they bear some meanings in other situations. Examples are 'terms' in 'in terms of' and 'well' in 'as well as'. We include some idioms of this type in the stop term list.

- $\quad$ Punctuations are used in determining stop terms. Some terms with punctuations before or after them can be known insignificant. Examples are 'first,', 'in particular,' and so on.

- The last category of stop terms is those expressions that provide meta-information. For example, business documents may contain 'Figure 1', 'Table 1' and so on. These expressions do not contribute to the description of document topics.

Other words in text that do not belong to stop terms are referred to as significant words. The stop term list expands traditional stop word list to a great extent. The current list includes 890 items, although it is not complete according to the above principles.

We studied the relationship between the number of significant words and the total number of words. Analysing the same samples used in the experiment in Section 4 of this paper, we found that there was a very strong linear relationship between the number of 
significant words and the total number of words as shown in Figure 1.

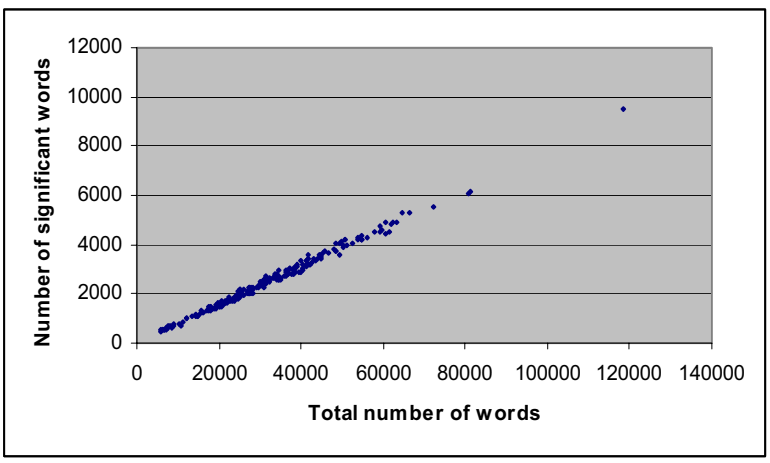

Figure 1. Relationship between the number of significant words and the total number of words

We conducted a linear regression analysis to determine the relationship and obtained the following equation

$$
\text { SigWd }=0.4953 T t \text { lWd }+42.8
$$

where SigWd is the number of significant words and $T t l W d$ is the total number of words. The analysis also shows that the correlation coefficient $R=0.99$, which indicates a very good linear relationship between the number of significant words and the total number of words. When this equation was used to estimate the number of significant words for the sample documents, the average relative error was merely $5.20 \%$. This result indicates that stop terms in the current list [20] account for about $50 \%$ of the total text. In other words, the amount of text processing can be reduced by $50 \%$ after the removal of the stop terms.

\subsection{Text segmentation}

Text segmentation will filter out certain words that seem irrelevant to the topics and sustain text segments that seem significant in representing the topics. For the text segmentation, the effort needed to implement and run complex linguistic methods in general is not worth the gain [21]. People do not always agree on where segment boundaries can be assigned [22]. According to our definition of the significant terms and stop terms, we assume that English text consists of significant terms that describe semantic topics of the text and stop terms that couple the significant terms. This assumption indicates a simple method of text segmentation.

The stop term list plays double roles in the text segmentation. Firstly, by referring to the list, we can remove stop terms to eliminate noises so that significant text can reduce to about $50 \%$ of its original size, according to our analysis in Section 3.1. Secondly, the stop terms can serve as delimiters of significant segments. Therefore we determine a significant segment by identifying its delimiters: stop terms and punctuations. The advantages of this method are twofold. Firstly, it is simple to locate the stop terms and punctuations, therefore, complex linguistic analysis is avoided. Secondly, the segment thus obtained retains a more complete and precise sense than words and phrases with a fixed number of words.

\subsection{Ordering terms}

We segment text for extracting keyterms to represent text topic. With our stop term list, we have obtained significant segments, which are the candidates of keyterms. Our task reduces to answer the question: how can we pick the most significant terms to describe the topic of a text, given that we know what the terms are, where they are and how frequently they occur in the text?

We followed the retrieval schemes proposed by [23] to rely on the geometric layout of documents. We assume that a document is presented at three levels of abstraction: the title, the abstract (or similar, for example the meta information in an HTML file) and the paragraphs that follow. Our method for keyterm extraction is based on occurrence frequencies of the significant terms, so we stress the role of a term at a higher level by assigning a higher weight to the frequency of the term. The weights are 5 for titles, 4 for abstracts and 1 for the paragraphs that follow. For example, the adjusted frequency of a term is 8 if it occurs twice in an abstract. Authors may create a new word by combining two words with a hyphen. A word with a hyphen is often significant to a topic discussed in the document, so we weight such a term with 1.5 to stress its significance.

One problem in this method is which term should be selected as a potential keyword among a phrase and its sub-phrase, for example, 'resource discovery discussed' and 'resource discovery'. As noun terms cannot be easily modified and repetition is notable for longer terms [22], we can reasonably predict that 'resource discovery' will occur more frequently than 'resource discovery discussed' in this example. It would be more appropriate to take 'resource discovery' as a single term. The phrase and sub-phrase should be counted as the same one. We select the most frequent one as their representative and the sum of their frequencies is taken as the frequency of the representative. In most cases, we can recognise the modifier of a term and determine the term appropriately in this way. 
We also consider the number of words in a phrase in the selection of keywords. Multiple-word phrases are more significant than single words, as a phrase often expresses a more definite meaning than a word. For example, 'information retrieval' is more definite in meaning than either 'information' or 'retrieval'. However, multiple-word phrases tend to cause matching difficulty in the retrieval process. In the English language, the chance that an $n$-word noun phrase (keyterms are normally nouns or noun phrases) is used becomes less with the increase of $n$ when $n>3$. Research also shows that 2-word phrases are the most useful type [7] and 5-word phrases do not provide additional benefit for IR [9].

We use the following formula to quantify the impact of $n$ on term significance.

$$
i(n)=0.25+\frac{2 \log _{10}(n)}{0.3 n}
$$

The graph of the formula is shown in Figure 2. The $i(1)=0.25$ is approximately $20 \%$ of $i(2) \approx 1.25$, which indicates that a phrase is much more significant in describing document contents than a single word. The $i(3) \approx 1.31$, which is about 0.06 greater than $i(2)$. With the increase of the $\mathrm{n}$, the $i(n)$ decreases gradually when $n>3$.

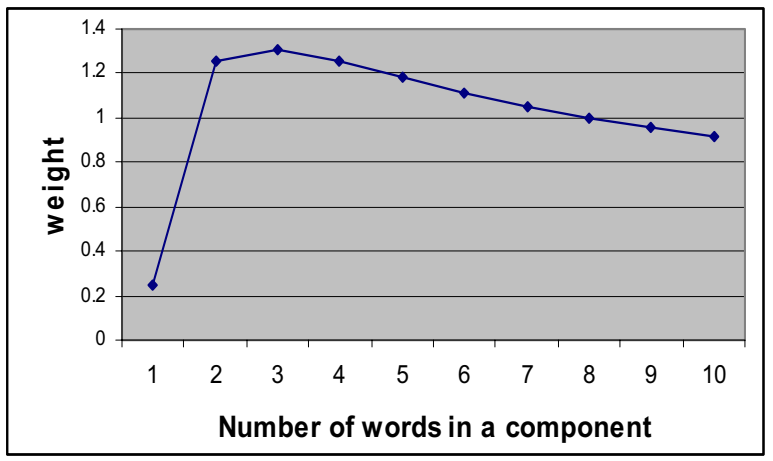

Figure 2 Impact of $\mathbf{n}$ on term significance

The rank index of a term is determined by the number of words in the term and its adjusted frequency $f:$

$$
r(n, f)=i(n) f=\left(0.25+\frac{2 \log _{10}(n)}{0.3 n}\right) f
$$

For any two terms that obtain the same rank score (both $n$ and $f$ are the same), the one that occurs earlier in the original document stands before the later one in the rank list. The top $m$ terms are selected as keyterms to describe the text contents.

In the keyterm selection scheme we considered the integrity tendency of noun terms, term position in the text, occurrence frequency of the term and the number of words in the term. With these considerations, we attempt to trim unnecessary modifiers from a term, judge the possibility that a term is significant in describing a document topic by its position and occurrence frequency, and select longer terms (by the number of words), which is more complete and definite in semantic meaning, as keyterms when other conditions are the same.

\section{Experimental evaluation}

To evaluate the proposed method of keyterm extraction, we downloaded sixty-six documents from the Web as a test sample. Each document included a set of keywords assigned by the author of the document. It was assumed that these keywords described the main topics of the document, and therefore they can be used for comparison with the extracted keyterms. The objective of the experiment was to test whether the method proposed in this research could extract as many of the keywords assigned by the author or semantically similar terms in the document. In the experiment, the keyword lists were removed from the sample documents, but kept as they were for the comparison with the experiment results.

Using the method discussed in Section 3, we obtained a ranked list of terms for each of the sixty-six documents. The number of unique terms in a list was from 338 to 1875 with an average of 1056 . We selected 20 top-ranked terms in each list and used them as the extracted terms that describe the topics of the document.

The extracted terms accounted for from $6 \%$ to $1 \%$ of the total number of unique terms in a document with an average of $2 \%$. For each document, we compared these 20 extracted terms with keywords assigned by the author of the document. The comparison results were categorised into four types:

1. Equal - a keyword indicated by the author is one of the extracted terms.

2. Component - a keyword is included in one or more of the extracted terms. For example, in Table 2, 'spatial' is included in 'erin spatial interface'.

3. Overlap - a part of a keyword is included in one or more of the extracted terms. For example, in Table 2, 'geographic information systems' overlaps included in 'query geographic information'.

4. Disjunction - no part of a keyword is included in any of the extracted terms.

The four types of the comparison result are illustrated in Figure 3 in next page. 


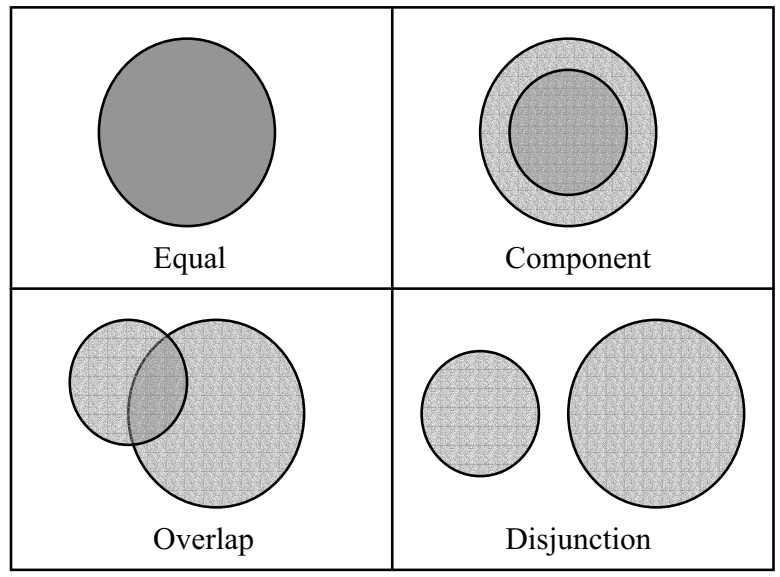

Figure 3. Venn's diagram of the four types of comparison result

Table 1 is a summary of the comparison. It shows that the extracted terms, which account for about $2 \%$ of the total terms, relate to $82 \%$ contents of the keywords. $57 \%$ of the keywords are included in the extracted terms.

Table 1. Comparison between extracted terms and keyword terms

\begin{tabular}{|c|c|c|}
\hline Type & $\begin{array}{c}\text { Number of } \\
\text { keywords }\end{array}$ & Percentage \\
\hline Equal & 96 & $30 \%$ \\
\hline Component & 86 & $27 \%$ \\
\hline Overlap & 78 & $25 \%$ \\
\hline Disjunction & 58 & $18 \%$ \\
\hline Total & 318 & $100 \%$ \\
\hline
\end{tabular}

These results are comparable with those of Kea, a keyphrase extraction system that uses a naïve Bayes learning method to induce a probabilistic model from the training corpus [24]. In a similar experiment, Kea averagely included about 1.30 to 1.55 "correct" keyphrases in 20 extracted keyphrases, depending on the four feature sets [25]. The method of this study averagely includes about 1.45 exact keywords assigned by authors in 20 extracted keyterms. Turney [25] compared Kea with GenEx, a keyphrase extraction system that consists of a set of parameterized heuristic rules tuned to training corpus by a genetic algorithm. Kea and GenEx achieve roughly the same level of performance. He also compared GenEx with C4.5 decision tree induction algorithm [26] and found that GenEx performs better than C4.5. The comparisons indicate that the simple method in this research study performs comparably well as or better than complex methods.

The result in Table 1 was obtained based on a simple morphologic comparison between extracted terms and keyword terms. Semantic analysis leads to a more optimal conclusion. We shall take an experiment case as an example to demonstrate semantic relationships of the terms. In this case, the disjunction occurs more than in other cases.

\section{Table 2. Semantic relationships between} extracted terms and keywords

\begin{tabular}{|c|c|}
\hline Rank No. & Extracted Term \\
\hline 1 & map interface \\
\hline 2 & $\begin{array}{l}\text { environmental resource information } \\
\text { network }\end{array}$ \\
\hline 3 & query geographic information \\
\hline 4 & map image \\
\hline 5 & vector file \\
\hline 6 & geographic region \\
\hline 7 & world wide web \\
\hline 8 & erin spatial interface \\
\hline 9 & $\begin{array}{l}\text { cgi (Common Gateway Interface) } \\
\text { script }\end{array}$ \\
\hline 10 & spatial data set directory \\
\hline 11 & mouse click \\
\hline 12 & minimum bounding rectangle \\
\hline 13 & environmental information \\
\hline 14 & project information \\
\hline 15 & region \\
\hline 16 & information require answer \\
\hline 17 & visual interactive access \\
\hline 18 & online form interface \\
\hline 19 & biological species distribution \\
\hline 20 & cape york peninsula \\
\hline $\begin{array}{c}\text { Related } \\
\text { Term No. }\end{array}$ & Keyword \\
\hline 8,10 & spatial \\
\hline 3,6 & geographic information systems \\
\hline 3,6 & gis (Geographic Information System) \\
\hline $3,6,8,10$ & geospatial \\
\hline 1,4, & map \\
\hline 2,13 & environmental \\
\hline 5 & cgm (Computer Graphics Metafile) \\
\hline 9 & scribble \\
\hline 2,13 & $\begin{array}{l}\text { erin (Environmental Resources } \\
\text { Information Network) }\end{array}$ \\
\hline 1,4, & imagemap \\
\hline 5 & vector \\
\hline $\begin{array}{l}2,3,7,13 \\
14,16\end{array}$ & search \\
\hline
\end{tabular}

In Table 2, extracted terms are listed in the order of their rank numbers. The keywords are related to the extracted terms by means of the rank numbers of the extracted terms. Extracted terms and keywords in bold belong to the disjunction category in the simple 
morphologic comparison. However, the keywords of this type relate semantically to one or more extracted terms that are relatively top-ranked. Therefore the extraction is effective. It is a subjective question whether the extracted terms in the disjunction category can join terms of other types to describe document contents more completely or simply bring noise, so we do not discuss these terms. For example, in Table 2, 'world wide web' and 'query geographic information' may imply 'query geographic information on the web'.

\section{Conclusion and future work}

Enterprise information is subject to frequent changes, and enterprise search calls for quick, simple and automatic indexing. The proposed method to extract keyterms from text meets this requirement of enterprise search. It automatically extracts important terms by simple text mining and avoids complex and lengthy linguistic and statistic process. It performs comparably to or better than other methods which are complex and need human intervention. As the extracted term list is ordered by weighting the terms, these terms with corresponding weights can be directly used for indexing purpose.

The proposed method for keyterm extraction can be used not only in automatic indexing, but also in query formulation, query expansion and generation of keyword summary of a document. As keyterms in one document are coherent to some extent, and documents in a Web site are also coherent to some extent, analysis of the association among keyterms of different documents will provide a means to solve the vocabulary problem. Further research work is needed for the application of the method in these aspects of IR.

\section{References}

[1] Hawking, D., Crimmins, F., Craswell, N. \& Upstill, T. 2004. How Valuable is External Link Evidence when Searching Enterprise Webs?. Fifteenth Australasian Database Conference (ADC2004), Dunedin, NZ, pp.77-84.

[2] Miller, T.W. 2005. Data and Text Mining: A Business Applications Approach. New Jersey: Pearson Prentice Hall.

[3] Mukherjee, R. \& Mao, J. 2004. Enterprise Search: Tough Stuff. ACM Queue, 2(2):37-46.

[4] Rao, R. 2004. From IR to search and beyond. $A C M$ Quеие, 2(3):67-73.

[5] Moulton, L. 2004. Understanding Taxonomies \& Search for Corporate Applications. The Gilbane Report, 12(4):2-12.

[6] Koster and Seutter, 2003. Taming wild phrases, Proceedings of the 25th European Conference on IR Research (ECIR 2003), Pisa, pp161-176.

[7] Pickens, J. and Croft, W.B. 2000. An exploratory analysis of phrases in text retrieval. RIAO 2000 Conference, Paris, pp1179-1195.
[8] Jiang, M., Jensen, E., Beitzel, S. \& Argamon, S. 2004. Effective use of phrases in language modelling to improve information retrieval, Symposium on AI \& Math Special Session on Intelligent Text Processing, Florida.

[9] Caropreso, M.F., Matwin, S. and Sebastiani, F. 2000. Statistical phrases in automated text categorization, Technical Report IEI-B4-07-2000, Istituto di Elaborazione dell'Informazione, Consiglio Nazionale delle Ricerche.

[10] Tseng, Y. 2002. Automatic Thesaurus Generation for Chinese Documents. Journal of the American Society for Information Science and Technology, 53(13):1130-1138.

[11] Zhou, L. \& Zhang, D. 2003. NLPIR: A Theoretical Framework for Applying Natural Language Processing to Information Retrieval. Journal of the American Society for Information Science and Technology, 54(2):115-123.

[12] Nakashima, M., Sato, K., Qu, Y. \& Ito, T. 2003. Browsing-Based Conceptual Information Retrieval Incorporating Dictionary Term Relations, Keyword Association, and a User's Interest, Journal of the American Society for Information Science and Technology, 54(1):1628.

[13] Yang, M., Yang, C.C. \& Chung, Y. 1997. A natural language processing based Internet agent. Proceedings of the IEEE International Conference on Multimedia Computing and Systems, Orlando, Florida, USA, pp.100-105.

[14] Baeza-Yates, R. \& Ribeiro-Neto, B. 1999, Modern Information Retrieval. Harlow, England: Addison Wesley.

[15] Greiff, W.R. 1999. Maximum entropy, weight of evidence and information retrieval. Department of Computer Science, University of Massachusetts.

[16] Strzalkowski, T. 1997. Robust text processing in automated information retrieval. Readings in Information Retrieval, Morgan Kaufman Publishers, pp317-322.

[17] Peñas, A. Gonzalo, J. \& Verdejo, F. 2001. Crosslanguage information access through phrase browsing. Proceedings of the 6th International Workshop on Applications of Natural Language to Information Systems, Madrid, pp121-130.

[18] Gao, X. \& Lo, B. 2001. Measuring document relevance in query space. Proceedings of the Asia Pacific Web Conference 2001, Changsha, China, pp.51-58.

[19] Gao, X., Murugesan, S. \& Lo, B. 2003. A Dynamic Information Retrieval System for the Web. Proceedings of the 27th International Computer Software and Applications Conference, Dallas, Texas, USA, pp. 663-667.

[20] Gao, X. 2004. A non-indexing approach to information retrieval. Thesis, School of Multimedia and Information Technology, Southern Cross University.

[21] Jones, K.S. 1999. What is the role of NLP in text retrieval?. In T. Strzalkowski, editor, Natural Language Information Retrieval, pp.1-24, Kluwer Academic, Dordrecht.

[22] Karlgren, J. 1999. The Basics of Information Retrieval, [On-line] Available at http://citeseer.nj.nec.com/200523.html. [23] Rus, D. \& Subramanian, D. 1997. Customizing Information Capture and Access. ACM Transactions on Information Systems, 15(1):67-101.

[24] Witten, I.H., Paynter, G.W., Frank, E., Gutwin, C. and Nevill-Manning, C.G. 2000. KEA: Practical automatic keyphrase extraction. Working paper 00/5, Department of Computer Science, The University of Waikato. 
[25] Turney, P.D. 2003. Coherent keyphrase extraction via Web mining. Proceedings of the 18th International Joint Conference on Artificial Intelligence (IJCAI-03), Acapulco, Mexico, pp.434-439.

[26] Turney, P.D. 1999. Learning to extract keyphrases from text, Technical Report ERB-1057, National Research Council, Institute for Information Technology. 